\title{
On the Analytical and Numerical Solutions of the One-Dimensional Non- linear Burgers' Equation
}

\author{
Talaat S. El-Danaf and Mohamed A. Ramadan*
}

Department of Mathematics, Faculty of Science, Menoufia University, Shebeen El-Koom, Egypt

\begin{abstract}
In this paper, we present two methods for finding solutions to the one-dimensional nonlinear Burgers' equation. The first method is an analytical one, which is based on the tanh - function method. The second method is based on the Adomian decomposition method where two approaches are introduced. The first approach considers the use of the initial condition while the other considers the use of the boundary conditions. The accuracy of the proposed numerical method is demonstrated by two test problems. The obtained results are presented and compared with the exact solution. The numerical results are found in good agreement with the exact solution in case of using the initial conditions and an acceptable accuracy for the case of using the boundary conditions. Finally, the results are presented graphically.
\end{abstract}

Keywords: Adomian decomposition Method, tanh -function Method, nonlinear Burgers' equation, analytical solution, accuracy.

\section{INTRODUCTION}

In this paper, we consider the one-dimensional nonlinear Burgers' equation in the form

$u_{t}+\varepsilon u u_{x}-v u_{x x}=0, \quad a \leq x \leq b$,

where; $\varepsilon, v$ are positive parameters and the subscripts $t$ and $x$ denote differentiation, with initial and boundary conditions:

$u(x, 0)=f(x)$

$u(a, t)=\beta_{1}, u(b, t)=\beta_{2} \quad \forall t>0$,

The study of Burgers' equation is important since it arises in the approximate theory of flow through a shock wave propagating in a viscous fluid and in the modeling of turbulence [1]. The exact solutions of Burgers' equation have been surveyed by Benton and Platzman [2]. In many cases these solutions involve infinite series which may converge very slowly or for small values of the viscosity coefficients. Several studies in the literature have been considred before to compute numerical solutions of Burgers'equation (see for example [3-5]).

In this paper, we are concerned first with introducing an analytical solution of Eq. (1) which is not of series form. Second, two approaches to approximate the solution of Eq. (1) based on the Adomian decomposition method (ADM) are proposed. The ADM is a numerical technique for solving linear or nonlinear partial differential equations by generating a functional series solution in a very efficient manner. It is closely related to the Taylor series method and fixed-point iteration method. It was shown [6-8] among others that the effectiveness of the ADM could be dramatically improved by determining further components of the solution $u(x, t)$.

*Address correspondence to this author at the Department of Mathematics, Faculty of Science, Menoufia University, Shebeen El- Koom, Egypt; Email:mramadan@mailer.eun.eg
The paper is organized as follows: In section 2, the use of tanh - function method [9-12] is demonstrated to get an analytical solution of Eq. (1). In section 3, an approximate solution is obtained by applying ADM using the initial condition $u(x, 0)=f(x)$ only. Then, a test example is given to demonstrate the accuracy of the method and to illustrate its pertinent feature. In section 4 , another approach for using ADM with the boundary conditions $u(a, t)=\beta_{1}, u(b, t)=\beta_{2}$ is proposed to get a numerical solution of Eq. (1), followed by a test example to demonstrate the accuracy of the method.

\section{ANALYTICAL SOLUTION USING THE TANH -} FUNCTION METHOD FOR BURGERS' EQUATION

In this section we find particular solutions for Burgers' equation (1) using the recent tanh-function method. For this, consider the transformations:

$u(x, t)=f(\xi)$,

where $\xi=c(x-\lambda t)$, where $c$ and $\lambda$ are arbitrary (real) constants. Based on this we use the following change of variables

$\frac{\partial}{\partial t}()=.-c \lambda \frac{d}{d \xi}(),. \frac{\partial}{\partial x}()=.c \frac{d}{d \xi}(),. \frac{\partial^{2}}{\partial x^{2}}()=.c^{2} \frac{d^{2}}{d \xi^{2}}($.

Applying the change of variable to Burgers' equation (1), the following ordinary differential equation is obtained.

$-c \lambda \frac{d f(\xi)}{d \xi}+\varepsilon c f(\xi) \frac{d f(\xi)}{d \xi}-c^{2} v \frac{d^{2} f(\xi)}{d \xi^{2}}=0$,

Integrating Eq. (5), we get

$-c \lambda f(\xi)+\frac{\varepsilon c}{2} f^{2}(\xi)-c^{2} v \frac{d f(\xi)}{d \xi}=B$,

where $B$ is the constant of integration.

Now we introduce a new independent variable:

$y=\tanh (\xi)$, 
that leads to the change of derivative

$\frac{d}{d \xi}()=.\left(1-y^{2}\right) \frac{d}{d y}($.

We introduce the following tanh series

$f(\xi)=s(y)=\sum_{i=0}^{m} a_{i} y^{i}$,

where $m$ is a positive integer. From Eqns. (8) and (9) we get

$-c \lambda s+\frac{c \varepsilon}{2} s^{2}--c^{2} v\left(1-\mathrm{y}^{2}\right) \frac{d s}{d y}=0$.

To determine the parameter $\mathrm{m}$ we balance the linear term of highest order in Eq. (10) with the highest order nonlinear term. This in turn gives $m=1$, so we get

$s(y)=a_{0}+a_{1} y$

Substituting $\mathrm{s}(\mathrm{y})$, and $s^{\prime}(y)$, from Eq. (11) into Eq. (10) yields the system of algebraic equations for $a_{0}, a_{1}, c$, and $\lambda$ :

$y^{0}:-c^{2} v a_{1}+0.5 c \varepsilon a_{0}^{2}-c \lambda a_{0}=0$,

$y^{1}: a_{0} a_{1} c \varepsilon-a_{1} c \lambda=0$,

$y^{2}: 0.5 \mathrm{c} \varepsilon \mathrm{a}_{1}^{2}+\mathrm{c}^{2} v \mathrm{a}_{1}=0$

with the aid of Mathematica we find two solutions:

$a_{0}=\frac{-2 c v}{\varepsilon}, a_{1}=\frac{-2 c v}{\varepsilon}, \lambda=-2 c v$,

$a_{0}=\frac{2 c v}{\varepsilon}, a_{1}=\frac{-2 c v}{\varepsilon}, \lambda=2 c v$,

So we obtain the solutions

$u(x, t)=\frac{2 c v}{\varepsilon}(-1-\operatorname{Tanh}[c(x+2 c v t])$,

$u(x, t)=\frac{2 c v}{\varepsilon}(1-\operatorname{Tanh}[c(x-2 c v t])$

which are solutions of Eq. (1).

In a similar way, the following solutions can be obtained

$u(x, t)=\frac{2 c v}{\varepsilon}(-1-\operatorname{Coth}[c(x+2 c v t])$,

$u(x, t)=\frac{2 c v}{\varepsilon}(1-\operatorname{Coth}[c(x-2 c v t])$

Remark: The obtained analytical solutions (14) and (15) for Burgers' equation (1) coincide with the one in [1]. This proves that tanh-function method can be applied easily to obtain analytical solution of some kinds of partial differential equations.

\section{THE ADM FOR BURGERS' EQUATION (USING THE INITIAL CONDITION)}

For the purpose of illustration of the methodology to the proposed method, using the ADM, we begin with considering Eq. (1) in the operator form:

$L u+R(u)+N(u)=g(t)$

where $L\left(L()=.\frac{\partial(.)}{\partial t}\right)$ is a linear operator and $\mathrm{R}$ is the remainder of the linear operator.
The nonlinear term is represented by $N(u)$. Thus we get

$L u=g(t)-R(u)-N(u)$

Assuming that the inverse operator $L^{-1}$ exists and it can be taken as the definite integral with respect to $t$ from $t_{0}$ to $t$, i.e.

$L^{-1}()=.\int_{t_{0}}^{t}() d$.

Then applying the inverse operator $\mathrm{L}^{-1}$ on both sides to Eq. (17) yields

$u=f_{0}+L^{-1}(g(t)-R(u)-N(u))$,

where $f_{0}$ is the solution of homogeneous equation

$L u=0$,

The integration constants involved in the solution of homogeneous equation (20) are to be determined by the initial condition $\left(u_{0}=u\left(x, t_{0}\right)=f(x),\right)$. The Adomian decomposition method assumes that the unknown function $u(x, t)$ can be expressed by a sum of components defined by the decomposition series of the form

$u(x, t)=\sum_{n=0}^{\infty} u_{n}(x, t)$,

with $u_{0}$ defined as $u(x, 0)$ where $u(x, t)$ will be determined recursively. The nonlinear operator in Eq. (16) can also be decomposed by an infinite series of polynomials given by

$N(\mathrm{u})=\sum_{\mathrm{n}=0}^{\infty} A_{n}$,

where $A_{n}$ are the appropriate Adomian's polynomials of $u_{0}, u_{1}, u_{2}, \ldots, u_{n}$ (that is $A_{n}\left(u_{0}, u_{1}, u_{2}, \ldots, u_{n}\right)$ defined by

$A_{n}=\frac{1}{n !} \frac{d^{n}}{d \lambda^{n}}\left[N\left(\sum_{i=0}^{\infty} \lambda^{i} u_{i}\right)\right]_{\lambda=0}=\frac{1}{n !}\left(\frac{d^{n}}{d \lambda^{n}}(f g)\right)_{\lambda=0}$

where

$f=\sum_{i=0}^{\infty} \lambda^{i} u_{i}=u_{0}+\lambda u_{1}+\lambda^{2} u_{2}+\lambda^{3} u_{3}+\ldots \ldots$

and $g=\sum_{i=0}^{\infty} \lambda^{i} u_{i x}=u_{0 x}+\lambda u_{1 x}+\lambda^{2} u_{2 x}+\lambda^{3} u_{3 x}+\ldots \ldots$

Simple calculations give us

$A_{n}=\frac{1}{n !}\left(\frac{d^{n}}{d \lambda^{n}}(f g)\right)_{\lambda=0}$,

$=\frac{1}{n !}\left[\left(D^{n} f\right) g+{ }^{n} C_{1}\left(D^{n-1} f\right)(D g)+{ }^{n} C_{2}\left(D^{n-2} f\right)\left(D^{2} g\right)+\ldots+f\left(D^{n} g\right)\right]_{\lambda=0}$

where ${ }^{n} C_{r}=\frac{n !}{r !(n-r) !}$ and $D^{n}=\frac{d^{n}}{d \lambda^{n}}$.

It is known in the literature that these polynomials can be calculated for all forms of nonlinearity according to algorithms constructed by Adomian [13,14] and recently developed by an alternative approach (see for example $[15,16]$ ). 
From the above analysis, the approximate solution of the nonlinear Burgers' equation is rewritten in the operator form Eq. (16) with the initial condition $u(x, 0)=u_{0}=f(x)$, can be determined by the series (23) with the iterative process:

$u_{0}(x, t)=f(x)$,

$u_{n+1}(x, t)=L^{-1}\left(g(t)-R\left(u_{n}\right)-A_{n}\right), n \geq 0$

Appling the inverse operator $\mathrm{L}^{-1}$ on both sides of Eq. (1) we get:

$u(x, t)=f(x)-L^{-1}\left(u u_{x}-v u_{x x}\right)$

Now, by using Eqns. (21), (22), (23) and (25) we get

$\sum_{n=0}^{\infty} u_{n}(x, t)=f(x)-L^{-1}\left(\sum_{n=0}^{\infty} A_{n}-v\left(\sum_{n=0}^{\infty} u_{n}\right)\right)$

Identifying the zero ${ }^{\text {th }}$ component $u_{0}(x, t)$ as $f(x)$, the remaining components $u_{n}(x, t), n \geq 1$ can be determined by using the recurrence relation (26). That is,

$u_{0}(x, t)=f(x)$

$u_{n+1}(x, t)=-L^{-1}\left(A_{n}-v\left(u_{n}\right)_{x x}\right), n \geq 0$

where $A_{n}$ are adomian's polynomials that represent the nonlinear term $\left(u u_{x}\right)$. One can see that the first few terms of $A_{n}$ are given by:

$A_{0}=u_{0 x} u_{0}$,

$A_{1}=u_{0 x} u_{1}+u_{1 x} u_{0}$,

$A_{2}=u_{0 x} u_{2}+u_{1 x} u_{1}+u_{2 x} u_{0}$,

$A_{3}=u_{0 x} u_{3}+u_{1 x} u_{2}+u_{2 x} u_{1}+u_{3 x} u_{0}$

The rest of polynomials can be generated in a similar way. The scheme in (27) can easily determine the components $u_{n}(x, t), n \geq 0$ and the first few components of $u_{n}(x, t)$ take the following form

$u_{0}(x, t)=f(x)$

$u_{1}(x, t)=-L^{-1}\left(A_{0}-v\left(u_{0}\right)_{x x}\right)$,

$u_{2}(x, t)=-L^{-1}\left(A_{1}-v\left(u_{1}\right)_{x x}\right)$,

$u_{3}(x, t)=-L^{-1}\left(A_{2}-v\left(u_{2}\right)_{x x}\right)$

$u_{4}(x, t)=-L^{-1}\left(A_{3}-v\left(u_{3}\right)_{x x}\right)$

Calculating more components in the solution series can enhance the numerical solution obtained by decomposition series. Consequently, one can recursively determine each individual term of the series $\sum_{n=0}^{\infty} u_{n}(x, t)$, and hence the solution $u(x, t)$ is readily obtained in a series form. For numerical purposes to test the accuracy of the proposed method, based on ADM, we consider two test cases for the Burgers' equation. The obtained numerical approximate solution for each case, $u_{a p p r}(x, t)$, is compared with the exact solution where

$u_{\text {appr. }}(x, t)=u_{0}(x, t)+u_{1}(x, t)+u_{2}(x, t)+u_{3}(x, t)+u_{4}(x, t)+\ldots$

\section{Test Case: 1}

Consider the following analytic solution of Burgers' equation [1]: $u(x, t)=\frac{1}{2}\left[1-\operatorname{Tanh}\left\{\frac{1}{4 v}\left(x-15-\frac{1}{2} t\right)\right\}\right], t \geq 0$

and the initial condition

$u(x, 0)=\frac{1}{2}\left[1-\operatorname{Tanh}\left\{\frac{1}{4 v}(x-15)\right\}\right]$,

where $x \in[0,30]$

Remark: This test problem has known initial conditions and applying ADM one needs initial conditions only the According to this example and the scheme in (29), we get:

$$
\begin{aligned}
& \mathrm{u}_{1}=\frac{0.0625 \mathrm{t}}{v}\left(1-\tanh ^{2}\left[\frac{1}{4 v}(\mathrm{x}-15)\right]\right) \\
& u_{2}=\frac{0.0078125}{v^{2}} t^{2} \operatorname{Sech}^{2}\left[\frac{1}{4 v}(x-15)\right] \operatorname{Tanh}\left[\frac{1}{4 v}(x-15)\right] \\
& \mathrm{u}_{3}=-\frac{\mathrm{t}^{3}}{3072 v^{3}} \operatorname{sech}^{2}\left[\frac{1}{4 v}(\mathrm{x}-15)\right] \\
& \left(1-3 \tanh ^{2}\left[\frac{1}{4 v}(\mathrm{x}-15)\right]\right)
\end{aligned}
$$

Or

$$
\begin{aligned}
& \mathrm{u}_{3}=\frac{\mathrm{t}^{3}}{3072 v^{3}} \operatorname{sech}^{4}\left[\frac{1}{4 v}(\mathrm{x}-15)\right] \\
& \left(-4+3 \cosh ^{2}\left[\frac{1}{4 v}(\mathrm{x}-15)\right]-\sinh ^{2}\left[\frac{1}{4 v}(\mathrm{x}-15)\right]\right)
\end{aligned}
$$

We obtain a numerical approximate solution for Burgers' equation. The obtained numerical results are summarized in Tables 1-4. From these results, we conclude that the proposed method, to calculate the approximate numerical solution of the Burgers' equation, gives remarkable accuracy in comparison with the exact solution for some values of time $t$.

Table 1. The Errors Between the Numerical and Exact Solutions for $\mathbf{t}=\mathbf{1}$ and $v=0.5$

\begin{tabular}{|c|c|c|c|}
\hline $\mathbf{x}$ & U Approximate & U Exact & Absolute error \\
\hline \hline 4 & 0.99998986 & 0.99998987 & $4.00932 \times 10^{-9}$ \\
\hline 8 & 0.99944700 & 0.99944722 & $2.13127 \times 10^{-7}$ \\
\hline 12 & 0.97068831 & 0.97068776 & $5.45743 \times 10^{-7}$ \\
\hline 16 & 0.37754702 & 0.37754066 & $6.35561 \times 10^{-6}$ \\
\hline 20 & 0.01098545 & 0.01098694 & $1.48505 \times 10^{-6}$ \\
\hline 24 & 0.00020339 & 0.00020342 & $3.48687 \times 10^{-8}$ \\
\hline 28 & $3.726 \times 10^{-6}$ & $3.7266 \times 10^{-6}$ & $6.4136 \times 10^{-10}$ \\
\hline
\end{tabular}

Table 2. The Errors Between the Numerical and Exact Solutions for $\mathbf{t}=\mathbf{3}$ and $v=0.5$

\begin{tabular}{|c|c|c|c|}
\hline $\mathbf{x}$ & U Approximate & U Exact & Absolute error \\
\hline \hline 4 & 0.99999543 & 0.99999627 & $8.39849 \times 10^{-7}$ \\
\hline 8 & 0.99975179 & 0.99979657 & $4.47788 \times 10^{-5}$ \\
\hline 12 & 0.98891035 & 0.98901305 & $1.02707 \times 10^{-4}$ \\
\hline 16 & 0.62015142 & 0.62245933 & $2.30791 \times 10^{-3}$ \\
\hline 20 & 0.02890743 & 0.02931223 & $4.04797 \times 10^{-4}$ \\
\hline 24 & 0.00054256 & 0.00055277 & $1.02176 \times 10^{-5}$ \\
\hline 28 & $9.9418 \times 10^{-6}$ & $1.013 \times 10^{-5}$ & $1.88157 \times 10^{-7}$ \\
\hline
\end{tabular}


Table 3. The Errors Between the Numerical and Exact Solutions for $\mathrm{t}=1$ and $v=0.4$

\begin{tabular}{|c|c|c|c|}
\hline $\mathbf{x}$ & U Approximate & U Exact & Absolute error \\
\hline \hline 4 & 0.99999942 & 0.99999942 & $7.6743 \times 10^{-10}$ \\
\hline 8 & 0.99991507 & 0.99991518 & $1.1337 \times 10^{-7}$ \\
\hline 12 & 0.98756129 & 0.98756834 & $7.0518 \times 10^{-6}$ \\
\hline 16 & 0.34871176 & 0.34864513 & $6.66311 \times 10^{-5}$ \\
\hline 20 & 0.00359200 & 0.00359360 & $1.5927 \times 10^{-6}$ \\
\hline 24 & 0.00002428 & 0.00002430 & $1.1512 \times 10^{-8}$ \\
\hline 28 & $1.6366 \times 10^{-7}$ & $1.6373 \times 10^{-7}$ & $7.7609 \times 10^{-11}$ \\
\hline
\end{tabular}

Table 4. The Errors Between the Numerical and Exact Solutions for $\mathrm{t}=3$ and $v=\mathbf{0 . 4}$

\begin{tabular}{|c|c|c|c|}
\hline $\mathbf{x}$ & U Approximate & U Exact & Absolute error \\
\hline \hline 4 & 0.99999968 & 0.99999983 & $1.5567 \times 10^{-7}$ \\
\hline 8 & 0.99995268 & 0.99997569 & $2.3012 \times 10^{-5}$ \\
\hline 12 & 0.99474997 & 0.99640639 & $1.6564 \times 10^{-3}$ \\
\hline 16 & 0.65542194 & 0.65135486 & $4.0670 \times 10^{-3}$ \\
\hline 20 & 0.01195175 & 0.01243165 & $4.7989 \times 10^{-4}$ \\
\hline 24 & 0.00008124 & 0.00008481 & $3.5667 \times 10^{-6}$ \\
\hline 28 & $5.4745 \times 10^{-7}$ & $5.715 \times 10^{-7}$ & $2.4048 \times 10^{-8}$ \\
\hline
\end{tabular}

The following figures (Figs. 1-12) show the behavior of the approximation solutions for the first test case.

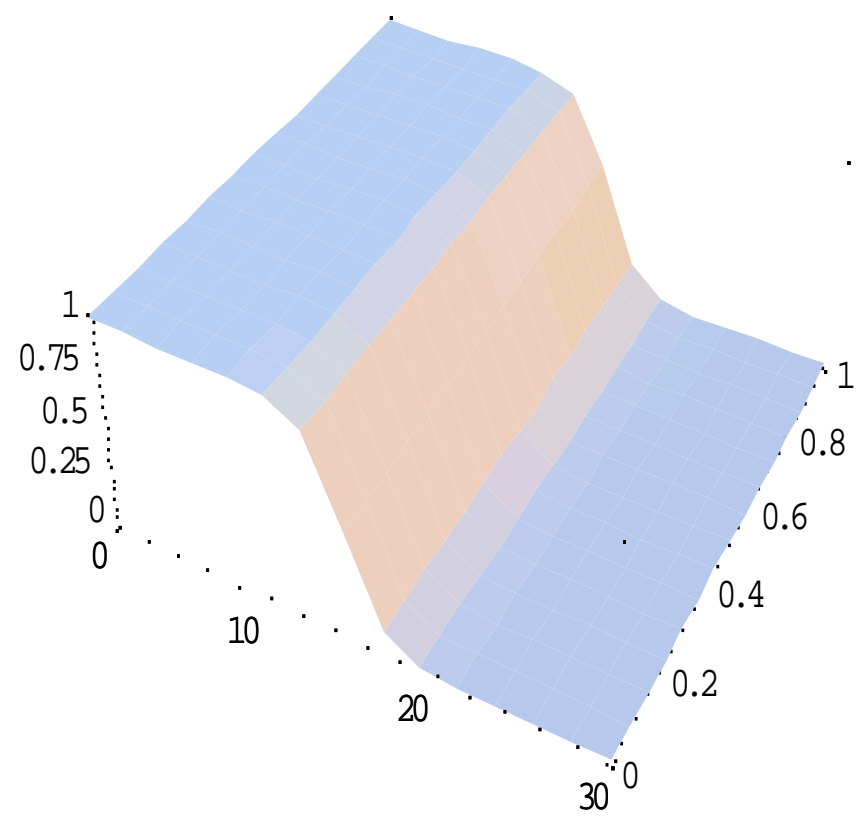

Fig. (1). The numerical solution ( $v=0.5$ from $t=0$ to $t=1$ ).

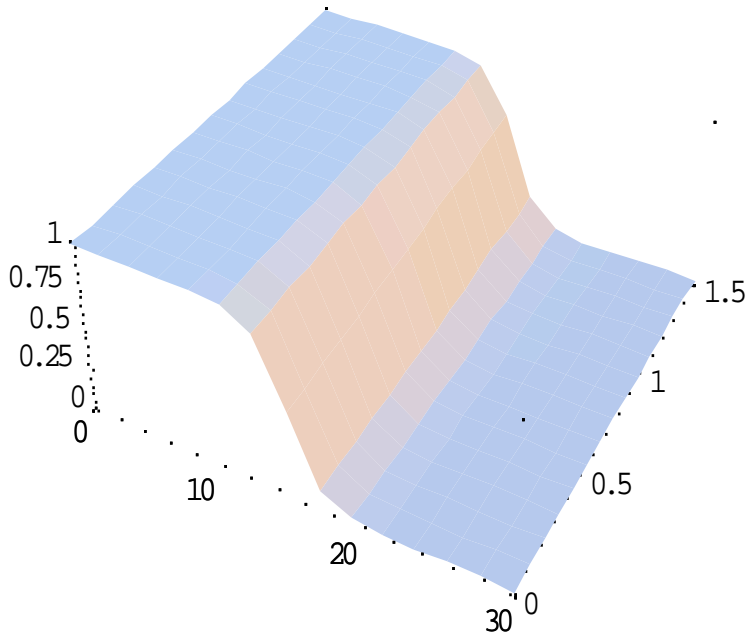

Fig. (2). The numerical solution ( $v=0.5$ from $\mathrm{t}=0$ to $\mathrm{t}=1.5)$.

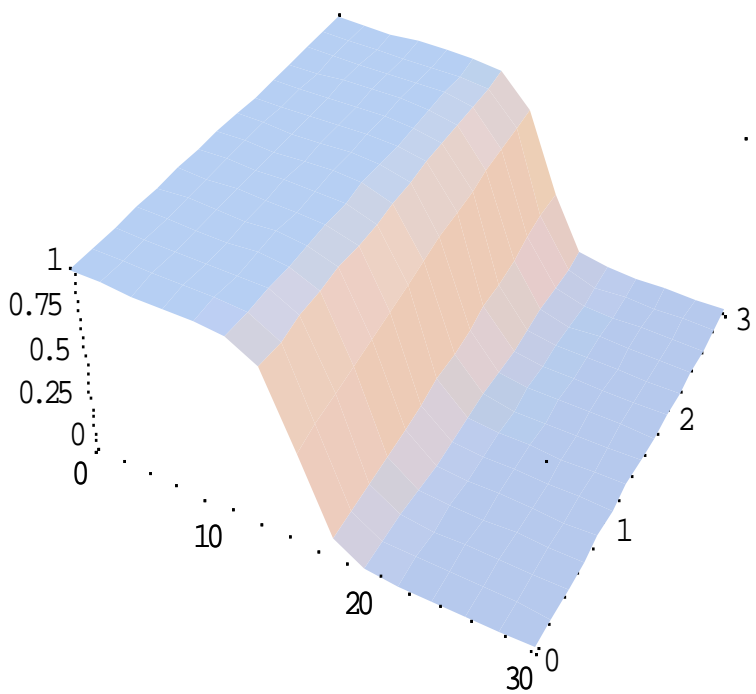

Fig. (3). The numerical solution ( $v=0.5$ from $\mathrm{t}=0$ to $\mathrm{t}=2$ ).

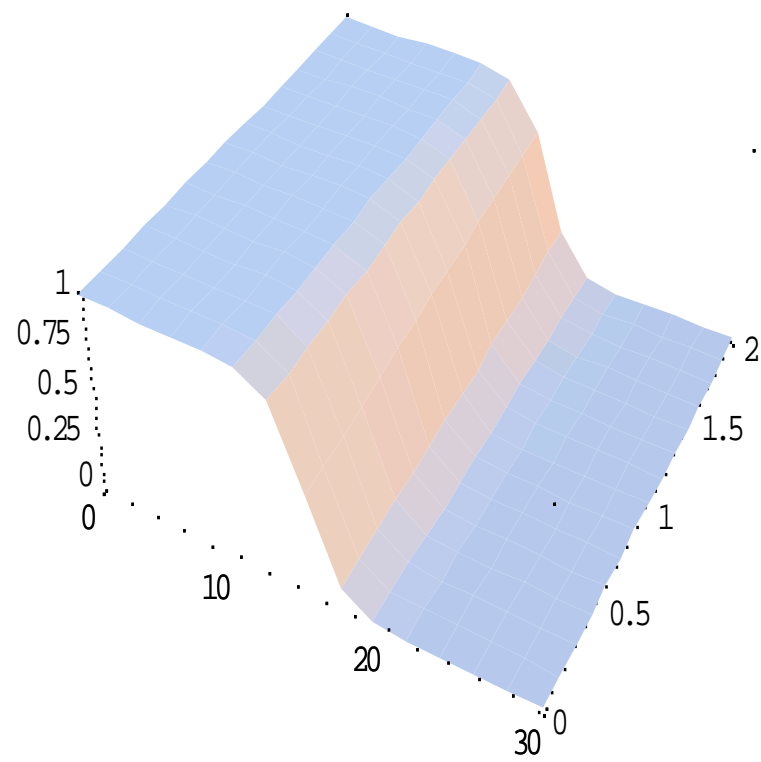

Fig. (4). The numerical solution ( $v=0.5$ from $\mathrm{t}=0$ to $\mathrm{t}=3.0$ ). 


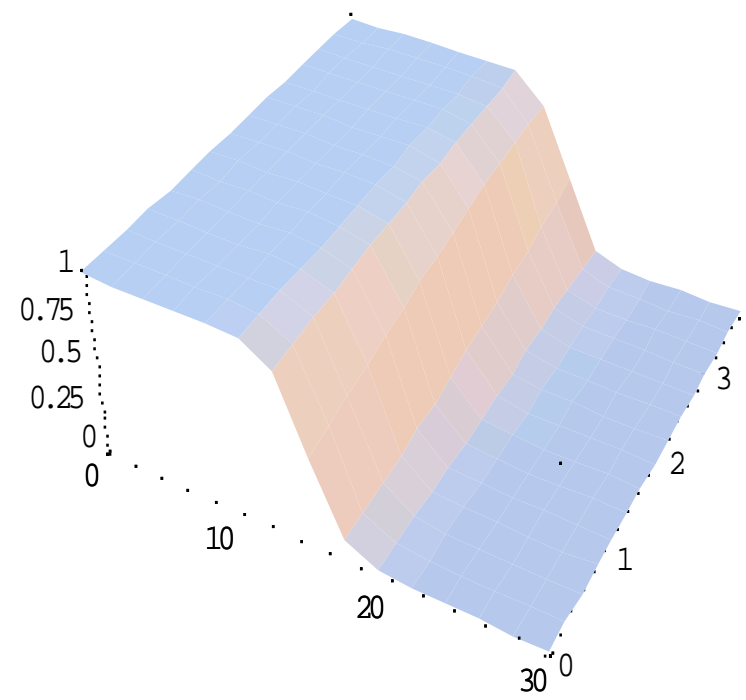

Fig. (5). The numerical solution ( $v=0.5$ from $\mathrm{t}=0$ to $\mathrm{t}=3.5)$.

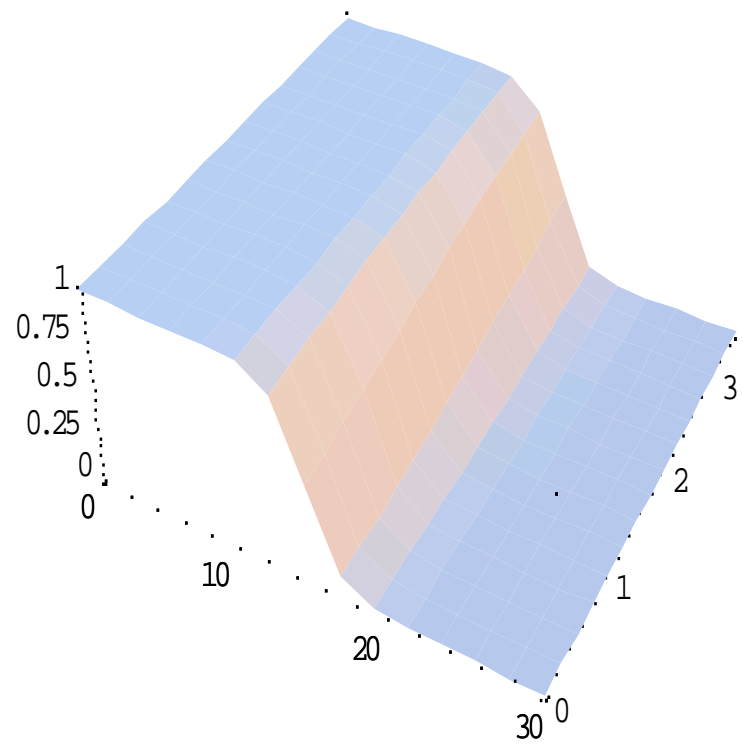

Fig. (6). The numerical solution ( $v=0.5$ from $\mathrm{t}=0$ to $\mathrm{t}=3.75$ ).

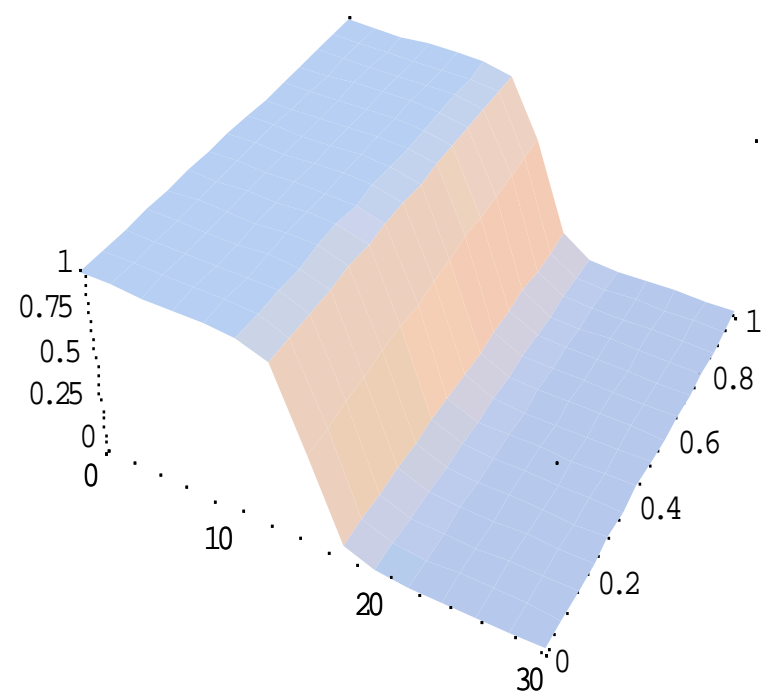

Fig. (7). The numerical solution ( $v=0.4$ from $\mathrm{t}=0$ to $\mathrm{t}=1$ ).

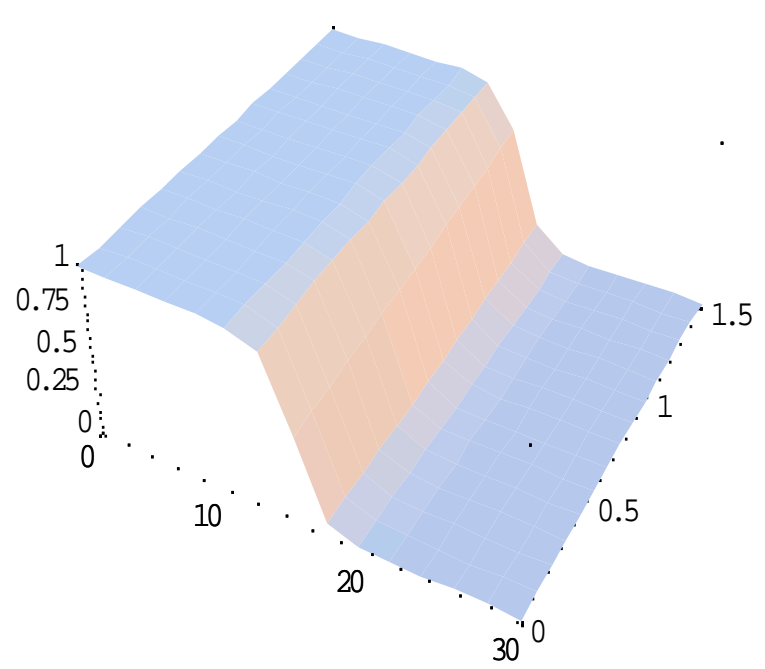

Fig. (8). The numerical solution ( $v=0.4$ from $\mathrm{t}=0$ to $\mathrm{t}=1.5$ ).

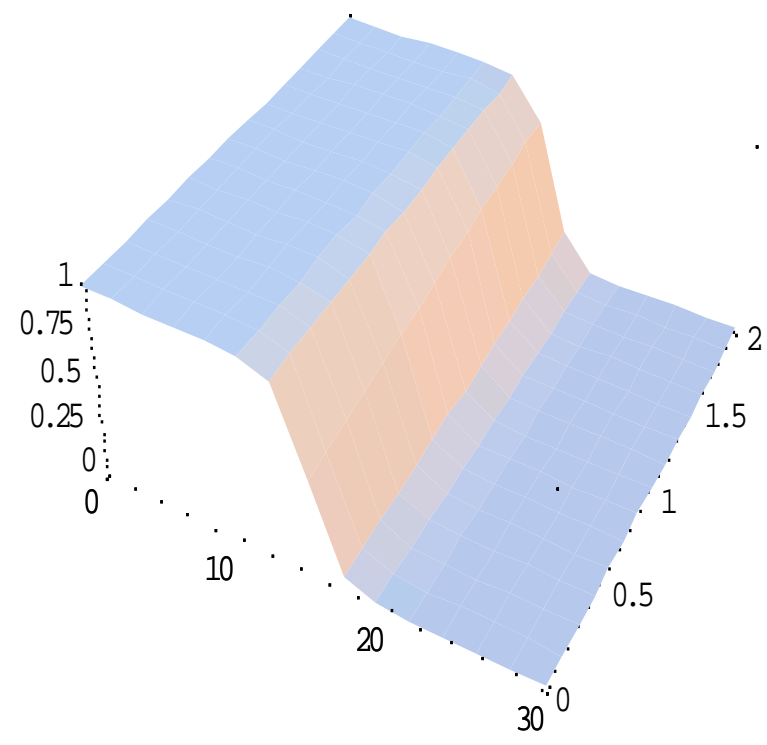

Fig. (9). The numerical solution ( $v=0.4$ from $t=0$ to $t=2$ ).

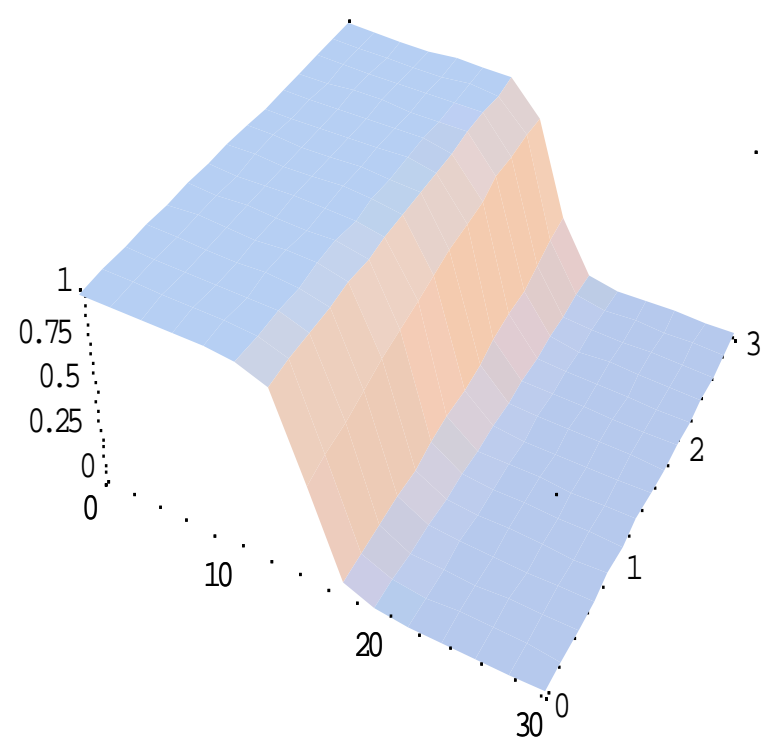

Fig. (10). The numerical solution ( $v=0.4$ from $t=0$ to $t=3.0$ ). 


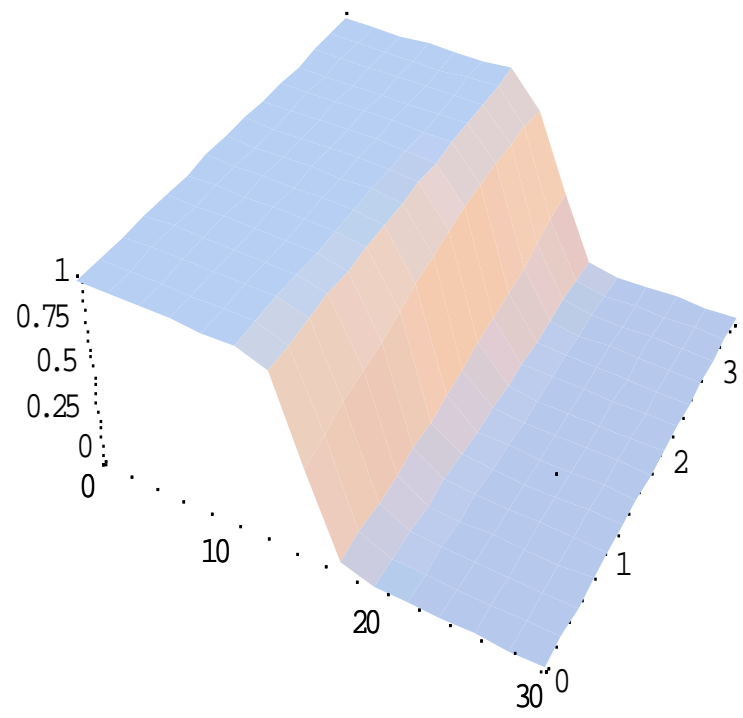

Fig. (11). The numerical solution ( $v=0.4$ from $t=0$ to $t=3.5$ ).

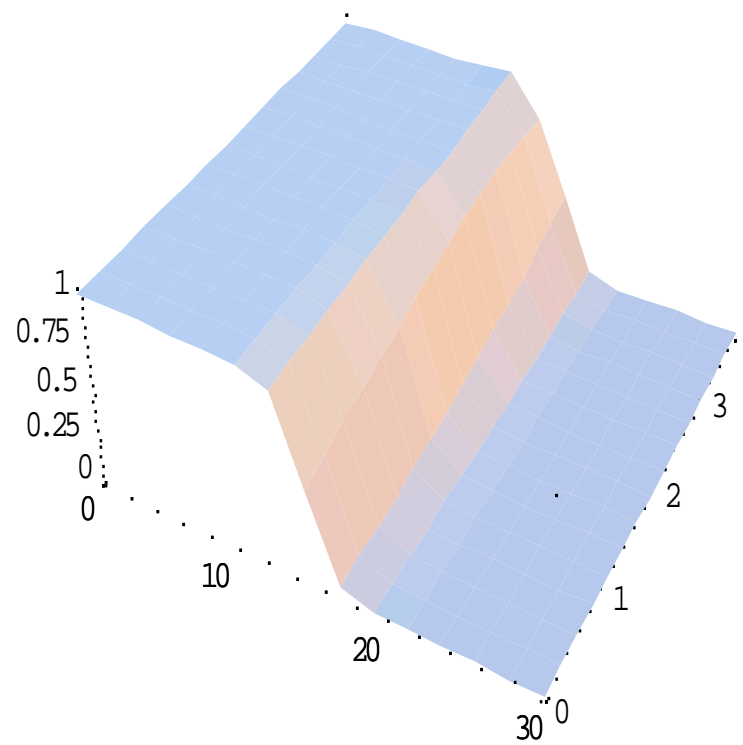

Fig. (12). The numerical solution $(v=0.4$ from $\mathrm{t}=0$ to $\mathrm{t}=3.75)$.

THE ADM FOR BURGERS' EQUATION (CONSIDERING THE BOUNDARY CONDITIONS)

Let $R=\frac{\partial^{2}}{\partial x^{2}}$. Then Eq. (1) can be expressed as

$R u=\frac{1}{v}\left[u_{t}+u u_{x}\right], \quad x \in[a, b]$

Applying the inverse operator $R^{-1}$ on both sides to Eq. (34) yields

$u(x, t)=\eta+\frac{1}{v} R^{-1}\left[u_{t}+u u_{x}\right]$

where $R^{-1}=\iint() d x d$,$x and \eta=C(t)+x B(t)$. Using Eqns. (21) and (22), Eq. (35) becomes

$\sum_{n=0}^{\infty} u_{n}(x, t)=\eta+\frac{1}{v} R^{-1}\left[\sum_{n=0}^{\infty} u_{n t}+\sum_{n=0}^{\infty} A_{n}\right]$ where $A_{n}=\sum_{m=0}^{n} u_{n-m} u_{m x}$. Now we decompose $\eta$ into $\eta=\sum_{n=0}^{\infty} \eta_{n}$ (double decomposition [3]). We have

$\sum_{n=0}^{\infty} u_{n}(x, t)=\sum_{n=0}^{\infty} \eta_{n}+\frac{1}{v} R^{-1}\left[\sum_{n=0}^{\infty} u_{n t}+\sum_{n=0}^{\infty} A_{n}\right]$

Identifying $u_{0}=\eta_{0}=C_{0}(t)+x B_{0}(t)$, all other components are determined by

$u_{n+1}=\eta_{n+1}+\frac{1}{v} R^{-1}\left[u_{n t}+A_{n}\right]$

where $\eta_{n+1}=C_{n+1}+x B_{n+1}, \quad n \geq 0$. The integration constants $C^{\prime}$ $s$ and $B$ ' $s$ are determined by satisfying the boundary conditions with the approximate solution $\phi_{n+1}=\sum_{k=0}^{n} u_{k}, n \geq 0$; Thus,

$\phi_{n+1}(a, t)=u(a, t)=\beta_{1}$

$\phi_{n+1}(b, t)=u(b, t)=\beta_{2}$

Our first approximation is $\phi_{1}=u_{0}$, or $\phi_{1}=C_{0}(t)+x B_{0}(t)$

Since $\phi_{1}(a, t)=u(a, t)=\beta_{1}, \phi_{1}(b, t)=u(b, t)=\beta_{2}$.

Therefore,

$C_{0}+a B_{0}=\beta_{1}$

$C_{0}+b B_{0}=\beta_{2}$

Solving (38) and (39), we get

$B_{0}=\frac{\beta_{2}-\beta_{1}}{b-a}$

$C_{0}=\frac{b \beta_{1}-a \beta_{2}}{b-a}$

Hence,

$u_{0}=\frac{(x-a) \beta_{2}+(b-x) \beta_{1}}{b-a}$

To calculate $u_{l}$, we have

$u_{1}=C_{1}+x B_{1}+\frac{1}{v} R^{-1}\left[u_{0 t}+A_{0}\right]$

A two- term approximation is given by

$\phi_{2}=\phi_{0}+u_{1}=u_{0}+u_{1}$

Hence,

$\phi_{2}=\frac{(x-a) \beta_{2}+(b-x) \beta_{1}}{b-a}+\frac{1}{v} R^{-1}\left[u_{0 t}+A_{0}\right]+C_{1}+x B_{1}$

Since $\phi_{2}(a, t)=\beta_{1}$ and $\phi_{2}(b, t)=\beta_{2}$, we have

$\zeta_{a}+C_{1}+a B_{1}=0$

$\zeta_{b}+C_{1}+b B_{1}=0$

where,

$\zeta_{a}=\frac{1}{v}\left[R^{-1}\left(u_{0 t}+A_{0}\right)\right]_{x=a}$ and $\zeta_{b}=\frac{1}{v}\left[R^{-1}\left(u_{0 t}+A_{0}\right)\right]_{x=b}$

Eqns. (43) and (44) give 
$B_{1}=\frac{\zeta_{a}-\zeta_{b}}{b-a}$

$C_{1}=\frac{a \zeta_{b}-b \zeta_{a}}{b-a}$

Using (45), (46) and (42), we get

$u_{1}=\frac{a \zeta_{b}-b \zeta_{a}}{b-a}+x\left(\frac{\zeta_{a}-\zeta_{b}}{b-a}\right)+\frac{1}{v} R^{-1}\left[u_{0 t}+A_{0}\right]$

We can continue in this manner to calculate $u_{2}, u_{3}, \ldots . .$.

\section{Test Case: 2}

The Burgers' equation has the analytic solution form [17]:

$u(x, t)=\frac{v}{1+v t}\left(x+\operatorname{Tan}\left[\frac{x}{2+2 v t}\right]\right), t \geq 0$,

and the boundary conditions with $\mathrm{a}=0.5, \mathrm{~b}=1.5$ gives

$\beta_{1}=u(0.5, t)=\frac{v}{1+v t}\left(\frac{1}{2}+\operatorname{Tan}\left[\frac{1}{4+4 v t}\right]\right)$,

$\beta_{2}=u(1.5, t)=\frac{v}{1+v t}\left(\frac{3}{2}+\operatorname{Tan}\left[\frac{3}{4+4 v t}\right]\right)$,

Using Eqns. (48) and (49); the two-term approximation $\phi_{2}=u_{0}+u_{1}$, we get the following results:

Remark: This test problem has known both initial and boundary conditions. For the technique applied in this case, we use the boundary conditions. Also, note that in general not all test problems have both initial and boundary conditions handy.

Table 5. The Errors Between the Numerical and Exact Solutions for $\mathrm{t}=\mathbf{2 . 1}$ and $v=1 / 500$

\begin{tabular}{|c|c|c|c|}
\hline $\mathbf{X}$ & $\phi_{2}=\mathbf{U}$ Approximate & $\mathbf{U}$ Exact & Absolute error \\
\hline \hline 0.6 & 0.00181349 & 0.00180833 & $5.16 \times 10^{-6}$ \\
\hline 0.8 & 0.00245054 & 0.00243143 & $1.911 \times 10^{-5}$ \\
\hline 1.1 & 0.0034405 & 0.00340559 & $3.491 \times 10^{-5}$ \\
\hline 1.4 & 0.00447673 & 0.00445588 & $2.085 \times 10^{-5}$ \\
\hline
\end{tabular}

Table 6. The Errors Between the Numerical and Exact Solutions for $\mathrm{t}=2.1$ and $v=1 / 50$

\begin{tabular}{|c|c|c|c|}
\hline $\mathbf{X}$ & $\phi_{2}=\mathbf{U}$ Approximate & U Exact & Absolute error \\
\hline \hline 0.6 & 0.0172417 & 0.0172003 & $4.14 \times 10^{-5}$ \\
\hline 0.8 & 0.0232607 & 0.0231078 & $1.529 \times 10^{-4}$ \\
\hline 1.1 & 0.032594 & 0.0323034 & $2.906 \times 10^{-4}$ \\
\hline 1.4 & 0.0422987 & 0.0421337 & $1.65 \times 10^{-4}$ \\
\hline
\end{tabular}

From Tables $\mathbf{5}$ and $\mathbf{6}$, we note for a small viscosity ( $v=1 / 500)$ that the numerical solution is very close to the exact solution.

Figs. (13-18) show the behavior of the numerical solution for some viscosity coefficients and various times.

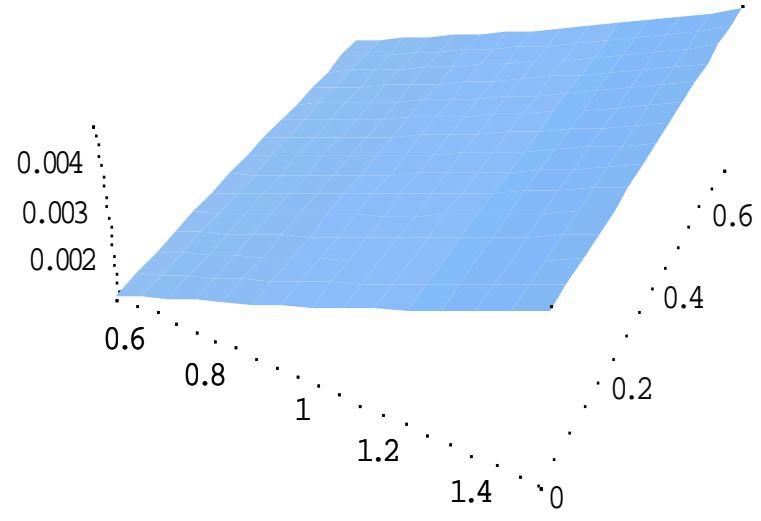

Fig. (13). The numerical solution ( $v=1 / 500$ from $\mathrm{t}=0$ to $\mathrm{t}=0.7$ ).

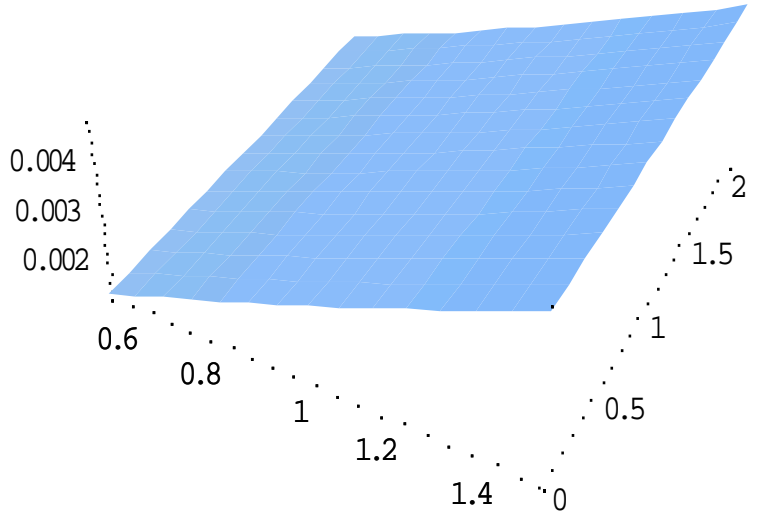

Fig. (14). The numerical solution ( $v=1 / 500$ from $\mathrm{t}=0$ to $\mathrm{t}=2.1)$.

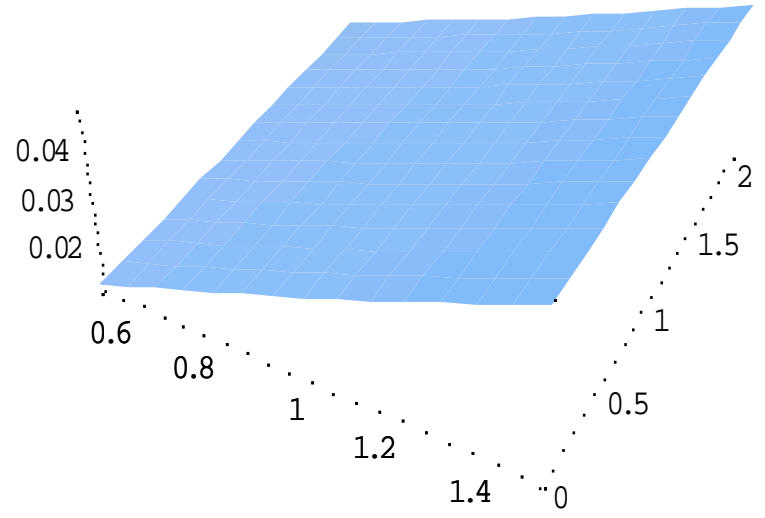

Fig. (15). The numerical solution ( $v=1 / 50$ from $\mathrm{t}=0$ to $\mathrm{t}=0.7$ ).

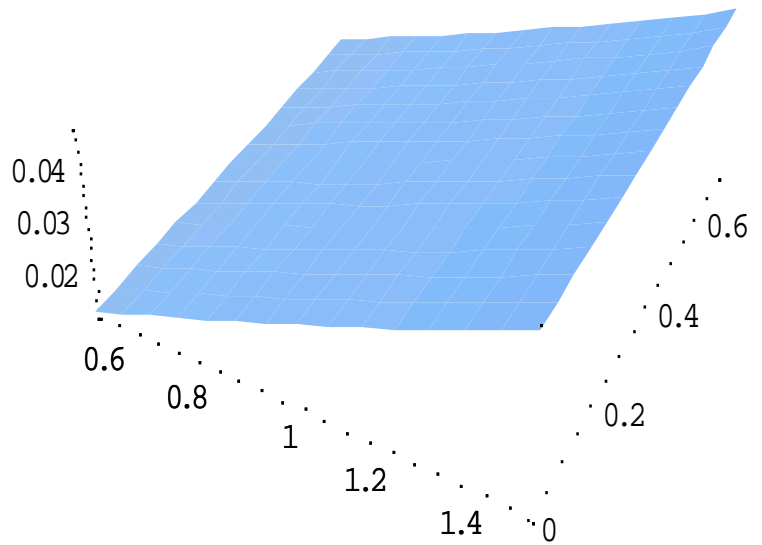

Fig. (16). The numerical solution ( $v=1 / 50$ from $\mathrm{t}=0$ to $\mathrm{t}=2.1)$. 


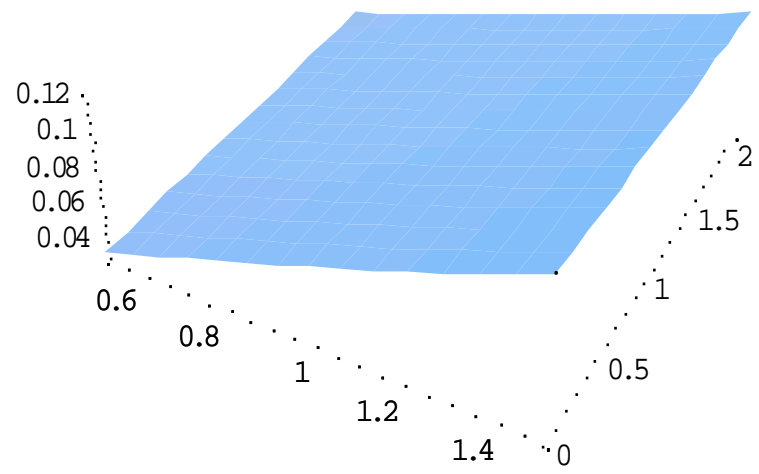

Fig. (17). The numerical solution $(v=1 / 20$ from $\mathrm{t}=0$ to $\mathrm{t}=0.7)$.

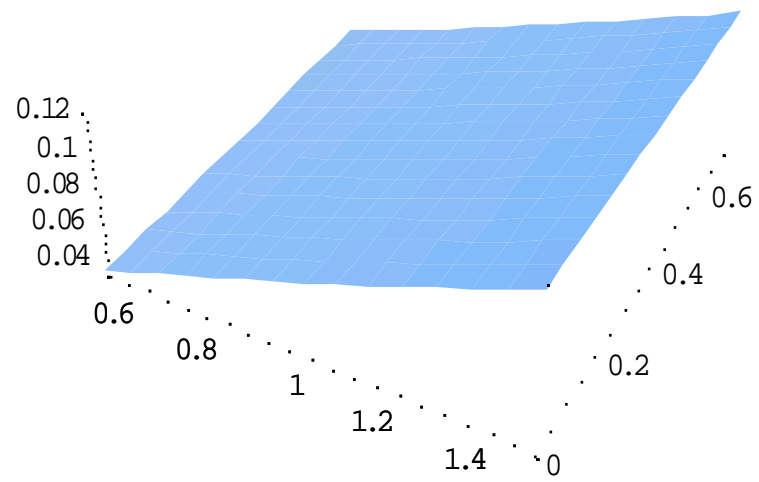

Fig. (18). The numerical solution $(v=1 / 20$ from $\mathrm{t}=0$ to $\mathrm{t}=2.1)$.

\section{CONCLUDING REMARKS}

In this paper, we considered analytical and numerical treatment for the solution of the Burgers' equation. From the theoretical analysis and the numerical results, we have the following remarks.

1. We can claim that tanh-function method is very successful for solving the Burgers equation.

2. The proposed tanh-function method is applicable for similar physical partial differential equations.

3. The obtained numerical results for Burgers' equation (1) showed that applying ADM with initial condition $u(x, 0)=f(x)$ provides remarkable accuracy, for various Reynolds number.
4. Although applying ADM with boundary conditions gives acceptable accuracy. The ADM in this case needs further study to improve the accuracy.

5. A Mathematica program is written easily and run on a PC Pentium IV, which takes a small run time.

\section{REFERENCES}

[1] Rubin SG, Graves RA. Viscous flow solutions with a cubic spline approximation. Computer and Fluids. 3,1-36 pergaman, 1975.

[2] Benton E, Platzman GW. A table of solutions of the one - dimensional Burger's equations. Quart Apl Math 1972; 30: 195-212.

[3] Ramadan MA, El Danaf TS. Numerical treatment for the modified Burger's equation. Math Comput Simul 2005; 70: 90-98.

[4] Ramadan MA, El Danaf TS, Abdel-Alaal FE. A numerical solution of the Burger's equation using septic B- splines. Chaos Solitons Fractals 2005; 26: 747-757.

[5] Dag I, Irk D, Saka B. A numerical solution of the Burger's equation using cubic spline Appl Math Comput 2005; 163: 199-211.

[6] Ismail HN, Raslan KR, Salem GS. Solitary wave solutions for the general $\mathrm{KdV}$ equation by adomian decomposition method. Appl Math Comput 2004; 154:17-29.

[7] Kaya D, El-Sayed SM. An application of the decomposition method for the generalized KdV and RLW equations. Chaos Solitons Fractals 2003; 17: 869-877.

[8] El-Danaf TS, Ramadan MA, Abdel-Alaal FE. Adomian decompostion method for solving the regularized long wave equation. Chaos Solitons Fractals 2005; 26: 747-757.

[9] Khater AH, Malfiet W, Callebaut DK, Kamel ES. The tanh method, a simple transformation and exact analytical solutions for nonlinear reaction-diffusion equations. Chaos Solitons Fractals 2002; 14: 513-522.

[10] Parkes EJ, Duffy BR. An automated tanh-function method for finding solitary wave solutions to nonlinear evolution equations. Comput Phys Commun 1998; 98: 288-300.

[11] Fan E. Extended tanh-function method and its applications to nonlinear equations. Phys Lett A 2000; 277: 212-218.

[12] Elwakil SA, El-labany SK, Zahran MA, Sabry R. Modified extended tanh- function method for solving nonlinear partial differential equations. Phys Lett A 2002; 299: 179-188.

[13] Adomian G. Solving Forntier problems of physics. The Decomposition Method. Kluwer, Boston: 1994.

[14] Adomian G. A review of the decomposition method in applied mathematics. J Math Anal Appl 1988; 135: 501-544.

[15] Wazwaz AM. A new Algorithm for calculating Adomian polynomials for nonlinear operators, Appl Math Comp 2000; 11: 33-51.

[16] Adomian G. New approach to non-linear partial differential equations. J Math Anal Appl 1984;102: 420-434.

[17] Mohsen A, Labib I. Some numerical experiments on the splitting of Burger's equation. Num Methods Part Differ Eqn 1992; 8: 267276. 